Artículo original

\title{
LA VALORACIÓN DEL PAISAJE URBANO Y SU RELACIÓN CON EL ESTADO DE CONSERVACIÓN DE LAZONA MONUMENTAL DE TACNA EN ELAÑO 2016.
}

The evaluation of the urban landscape and its relation with the state of conservation of the monumental zone of Tacna in the year 2016.

Renza Lourdes Gambetta Quelopana ${ }^{1}$

\section{RESUMEN}

El objetivo de la investigación es establecer la relación entre el nivel de valoración con el nivel del estado de conservación en la Zona Monumental de Tacna en el año 2016. Investigación de tipo aplicada y cuantitativa, descriptiva y correlacional. El instrumento fue confeccionado a partir de modelos aplicados por los Municipios españoles, los cuales se basan a su vez en el Modelo Europeo de Paisaje. Validado por juicio de expertos. Se utilizó el coeficiente de correlación lineal de Pearson para identificar la correlación entre las variables analizadas. Los resultados reflejan una relación entre la variable valoración y la variable estado de conservación, aplicado al caso de estudio de la Zona Monumental de Tacna. Se concluyé que existe una relación directa y significativa entre la valoración del paisaje urbano y el estado de conservación de la Zona Monumental de Tacna en el año 2016 (Correlación de Pearson de 0.936 y valor de significancia del 0.01).

Palabras clave: correlación, relación directa, valoración, estado de conservación.

\section{ABSTRACT}

The objective of the research is to establish the relationship between the level of assessment and the level of conservation status in the Tacna Monumental Zone in 2016. Research applied and quantitative, descriptive and correlational. The instrument was made from models applied by the Spanish Municipalities, which are based in turn on the European Landscape Model. Validated by expert judgment. Pearson's linear correlation coefficient was used to identify the correlation between the variables analyzed. The results reflect a relationship between the valuation variable and the conservation status variable, applied to the case study of the Tacna Monumental Zone. It was concluded that there is a direct and significant relationship between the assessment of the urban landscape and the state of conservation of the Tacna Monumental Zone in 2016 (Pearson correlation of 0.936 and significance value of $0.01)$

Key words: correlation, direct relation, valuation, state of conservation.

\footnotetext{
1 Magíster en Arquitectura Paisajista
}

Docente de la Universidad Privada de Tacna, Coordinadora CIAM -CPAR

\section{INTRODUCCIÓN}

Tacna es una ciudad que ha crecido en diferentes etapas en el transcurso del tiempo, con diferentes momentos de ocupación que se remontan a los primeros pobladores en el período pre hispánico, pasando luego por el período colonial y republicano hasta la actualidad. Uno de los momentos más significativos en la historia de Tacna lo podemos encontrar en la etapa comprendida entre la guerra del Pacífico, el período del cautiverio y el retorno de Tacna al Perú. Este período es singularmente valioso ya que se identifican momentos significativos que han dejado huella en el paisaje urbano de la ciudad, lo cual forma parte de la identidad y la memoria colectiva de los pobladores y visitantes de Tacna. El sector más representativo de la ciudad, en la que podemos identificar la mayor parte de elementos significativos se ubica en el centro de la ciudad, lo que hoy es denominado la Zona Monumental de Tacna. El conocimiento del valor del paisaje urbano y la relación que éste tiene con su estado de conservación permitirá mejorar la comprensión, manejo del mismo y servir para aportar a la implementación de instrumentos de manejo.

El paisaje atraviesa hoy una situación paradójica y crítica. La sociedad cosmopolita occidental que, en estos momentos, admira, ama, teoriza y piensa sobre sus paisajes más que en ningún otro momento de su historia, destroza y devora sus paisajes más que nunca(Ojeda, J., \& Cano, N., 2009). El paisaje, es a la vez, una realidad física y la representación que culturalmente nos hacemos de ella; la fisionomía externa y visible de una determinada porción de la superficie terrestre y la percepción individual y social que genera. Un tangible geográfico y su interpretación intangible. Es al mismo tiempo, significante y significado, el continente y el contenido, la realidad y la ficción (Nogué i Font, J., 2007). La Zona Monumental es un sector de la ciudad cuya fisonomía debe conservarse porque poseen valor urbanístico de conjunto, poseen valor documental históricoartístico y en ellas se encuentra un número apreciable de monumentos y/o ambientes urbanos monumentales. La delimitación de este sector urbano estuvo a cargo del Ministerio de Cultura, quienes a través de un estudio determinaron reducir significativamente el área declarada como tal a través de la Resolución Ministerial Nº 0928-80-ED, 
publicada en el año 1980, generándose una nueva delimitación mediante la Resolución Vice Ministerial $N^{\circ}$ 138-2014-VMPCIC-MC de fecha 22 de diciembre de 2014. Según el Convenio Europeo "paisaje" se entenderá cualquier parte del territorio tal como la percibe la población, cuyo carácter sea el resultado de la acción y la interacción de factores naturales y/o humanos(Europeo, E. m., 2000). El paisaje cultural es una realidad dinámica, resultado de los procesos que se producen a lo largo del tiempo en un territorio, y compleja porque la integran componentes naturales y culturales, materiales e inmateriales, tangibles e intangibles (Gómez Villarino, A., 2012). Según la UNESCO (UNESCO, 2006) define como paisaje a lo claramente definido, creado y diseñado intencionadamente por el ser humano aquellos ajardinados y parques, construidos por razones estéticas, evolucionado orgánicamente, debido a un imperativo inicial de carácter social, económico, administrativo y/o religioso, y que ha evolucionado hasta su forma actual como respuesta a la adecuación a su entorno natural, con los subtipos: paisaje vestigio (o fósil), paisaje activo, y paisajes culturales. El paisaje urbano está referido fundamentalmente a la imagen de un área o territorio determinado, ya sea rural, urbano, acuático, atmosférico, o a una situación combinada entre estos. Es aquel que significa el mayor grado de transformación de los recursos y paisajes naturales. Expresa el mayor grado de transformación de los recursos y paisajes naturales. Es un fenómeno físico que se modifica permanentemente a través de la historia y paralelamente con el desarrollo de la ciudad(Pérez, E., 2000). El estudio de paisaje es un instrumento de dinamización y de mejora de la calidad del territorio y una herramienta muy útil para orientar los futuros desarrollos urbanísticos y territoriales, preservando la identidad de cada lugar y contribuyendo a la funcionalidad de la infraestructura verde del territorio (Muñoz Criado, A., 2012). Las unidades de paisaje se centran en delimitar porciones de territorio con cierto grado de homogeneidad, entendiendo que dicha homogeneidad debe comprenderse de forma gradual y de acuerdo a determinados parámetros de referencia (Serrano Giné, D., 2014).Se entiende por recursos paisajísticos los elementos lineales o puntuales singulares de un paisaje que definen su individualidad y que tienen un valor visual, ecológico, cultural y/o histórico(Comunidad Valeciana, 2016). Desde el punto de vista de la ética, la valoración se refiere a un determinado juicio que cada acto tiene a los ojos de un individuo o de la sociedad(Definición MX, s. f.). El territorio está integrado por elementos naturales o artificiales los cuales conforman las cualidades visuales intrínsecas. El relieve posee múltiples elementos capaces de dar una respuesta visual diferente por su color, lo destacado de las formas, por su textura - rugosidad, etc. La hidrografía, aunque no presentan la importancia de otras coberturas continuas que aportan a la estructura básica, pueden tener una importancia considerable por ser un elemento de contraste y diversidad visual(Másmela Díaz, Pamela, 2010). Los elementos bióticos están conformados por la vegetación y los componentes antrópicos que corresponden a las actuaciones humanas como las actividades agrícolas y ganaderas; las obras públicas; la industria y la minería; la urbanización y edificaciones; y las actividades turísticas y deportivas(Másmela Díaz, Pamela, 2010). El estado de conservación o estado de deterioro es el registro de los deterioros que presenta un objeto en un momento determinado de observación (Instituto Nacional de Cultura, 2009). Se entiende por calidad de un paisaje "el grado de excelencia de éste, su mérito para no ser alterado o destruido, o de otra manera, su mérito para que su esencia y su estructura actual se conserve"(Blanco, A. A., 1979). El paisaje como cualquier otro elemento tiene un valor intrínseco, y su calidad se puede definir en función de su calidad visual intrínseca y del horizonte escénico que lo enmarca(Cifuentes, P., 1979). Se considera como un elemento del medio físico más a tener en cuenta como clave ambiental donde surge la necesidad de llevar a cabo una valoración (Ormaetxea Arenaza, O., 1997). Esta evaluación se valora directamente de modo subjetivo, utilizando calificativos, escalas de rango o de orden (Muñoz-Pedreros, 2004).

\section{OBJETIVOS}

- Identificar el nivel de valoración del paisaje urbano en la Zona Monumental de Tacna.

- Valorar el estado de conservación del paisaje urbano de la zona monumental de Tacna.

\section{METODOLOGÍA}

La investigación se desarrolla en el ámbito de la Zona Monumental de Tacna, Perú entre enero de 2016 a enero de 2017. Investigación no experimental de corte transversal, en el nivel relacional. La población, objeto de estudio, estuvo constituida por 70 personas considerados expertos o conocedores del ámbito de estudio. Se utilizó una Escala de medición donde se considera la Valoración y Estado de Conservación monumental con un puntaje máximo de 5 puntos y mínimo de 1 puntos basados en la escala Likert. El Instrumento fue confeccionado en base a basada en la Encuesta de Valoración ciudadana del paisaje del 
Municipio de Facheca, Diputación de Alicante, y del cuestionario de valoración paisajística que forma parte del estudio de integración paisajística del proyecto de drenaje integral de la rambla de Alcalà, incorporando, al mismo, los tramos II, III Y IV del T.M. de Benicarló (Ministerio de Medio Ambiente y medio Rural y Marino., 2008), con niveles para las variables calidad del servicio y satisfacción del cliente de 5 : Muy alto; 4: Alto; 3: Regular; 2: Bajo; 1: Muy bajo. El instrumento considera 27 preguntas tanto para la escala de valoración como para la variable estado de conservación, con valores que oscilan entre 0 y 135 puntos. Para contrastar los resultados se utilizó el coeficiente de Correlación de Pearson.

\section{RESULTADOS}

Según el indicador Calidad Visual Intrínseco se aprecia que el $38 \%$ de la muestra establece que es alta y un $31 \%$ le parece muy alta. Se valora de manera positiva la calidad visual de la Zona Monumental de Tacna. Ninguno de los encuestados opinó con valoración muy baja (graf. 1).

En lo que respecta a el indicador de Visibilidad, el $30 \%$ de la muestra establece que es alta, un $29 \%$ le parece baja y un $26 \%$ opina que la visibilidad es media (Gráf. 2).

Graf. 1: Calidad visual intrínseca

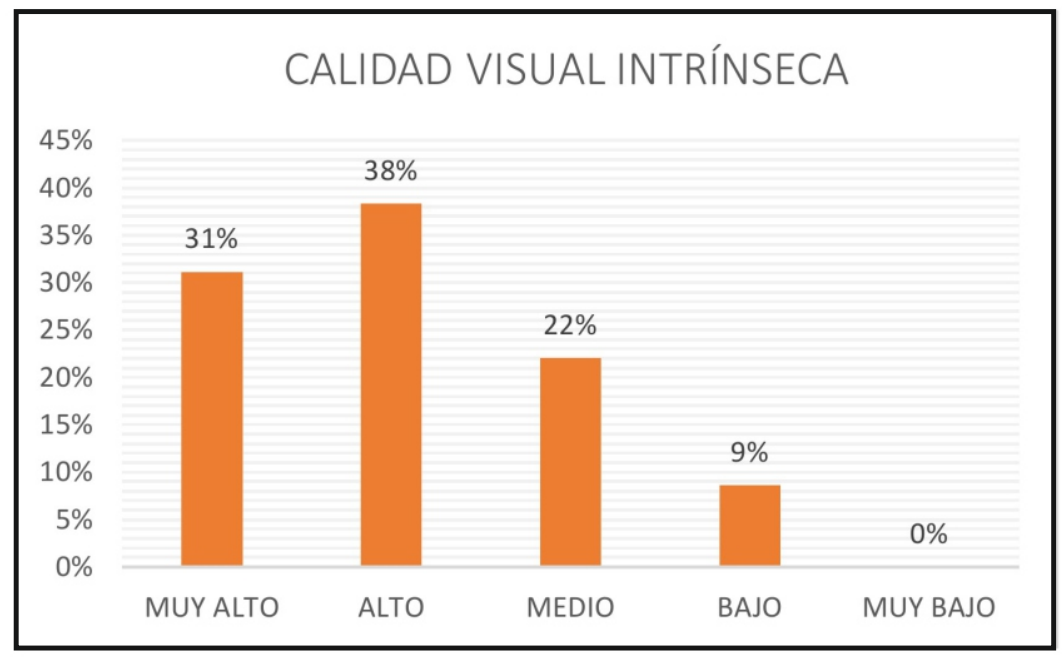

Graf. 2: Visibilidad

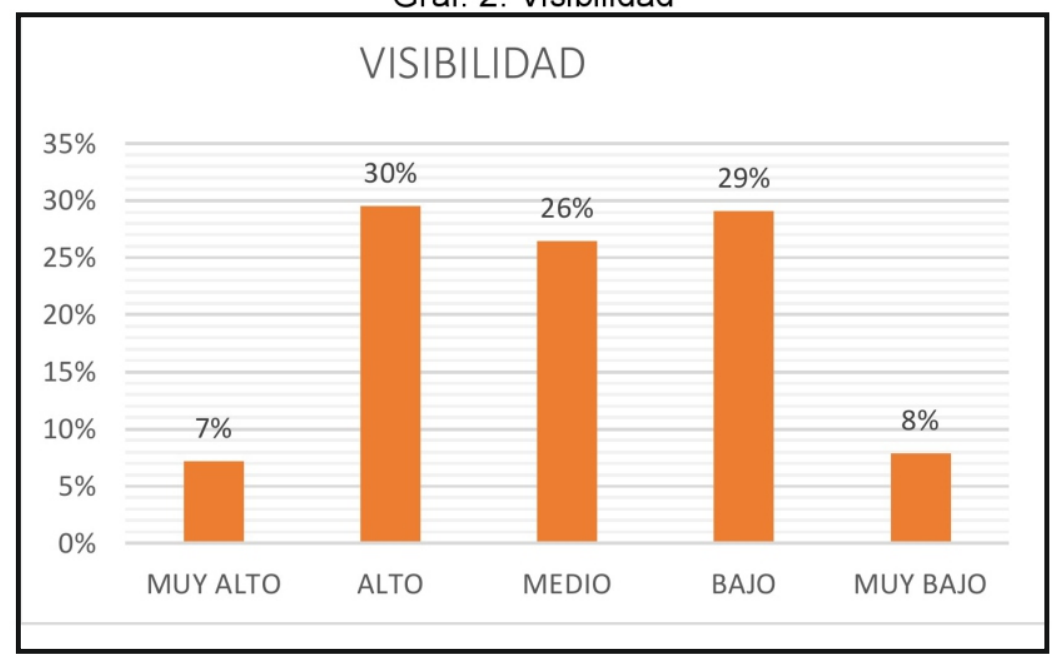

Según el indicador Recursos Paisajísticos de la Unidad de Paisaje 01, se observa que $40 \%$ de la muestra establece que es muy alta, un $34 \%$ le parece alta y un $20 \%$ opina que la valoración de los recursos paisajísticos de este sector es media (Graf. 03). En referencia al indicador Recursos Paisajísticos de la Unidad de Paisaje 02, el 37\% de la muestra establece una valoración medio, un $29 \%$ le parece alta y un $26 \%$ opina que la valoración de los recursos paisajísticos de este sector es baja (Graf. 04). En el indicador Recursos Paisajísticos de la Unidad de Paisaje 03, el 
$37 \%$ de la muestra establece una valoración medio, un 31\% le parece baja y un 30\% opina que la valoración de los recursos paisajísticos de este sector es media (Graf. 5)

Graf. 3: Recursos paisajísticos UP 01

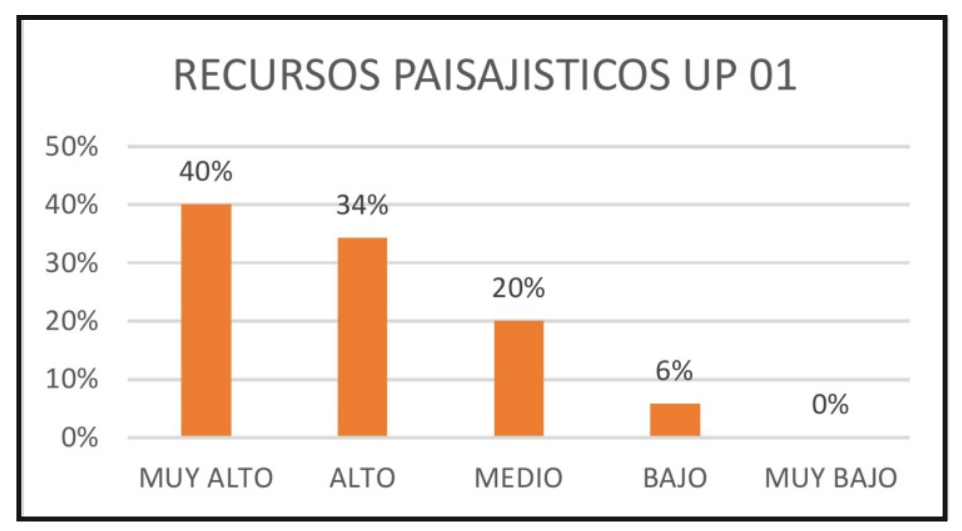

Graf. 4: Recursos paisajísticos UP 02

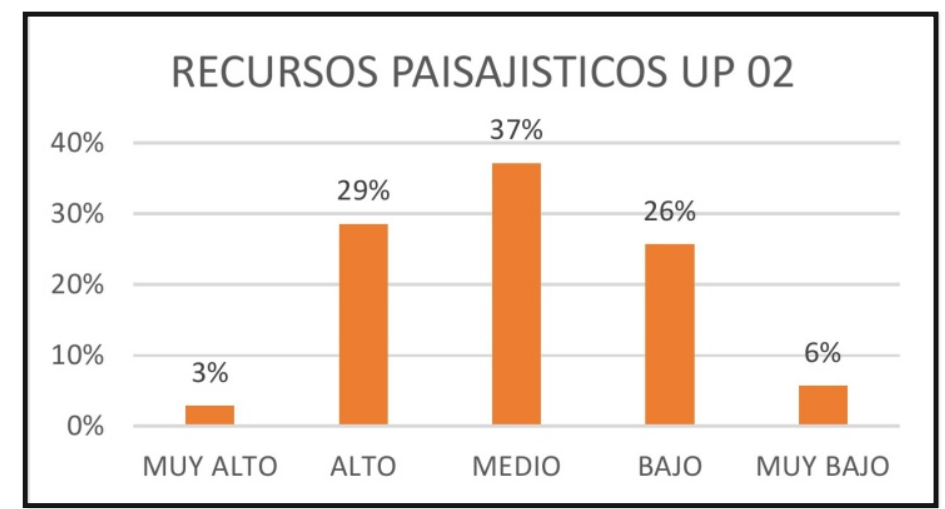

Graf. 5: Recursos paisajísticos UP 03

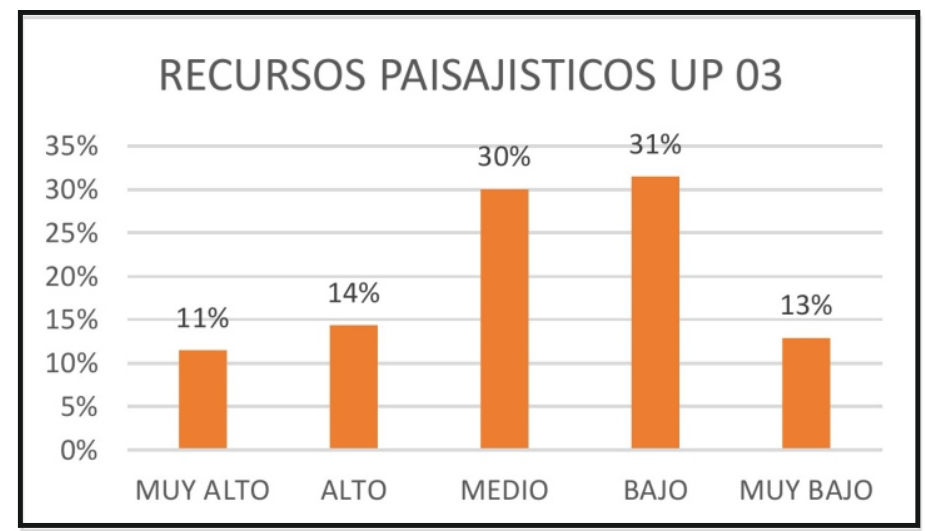

Según el indicador Recursos Paisajísticos, se aprecia que el 30\% de la muestra establece una valoración medio, un $26 \%$ la considera alta y un $17 \%$ la considera muy alta (Graf. 06) 
Graf. 06: Recursos Paisajísticos.

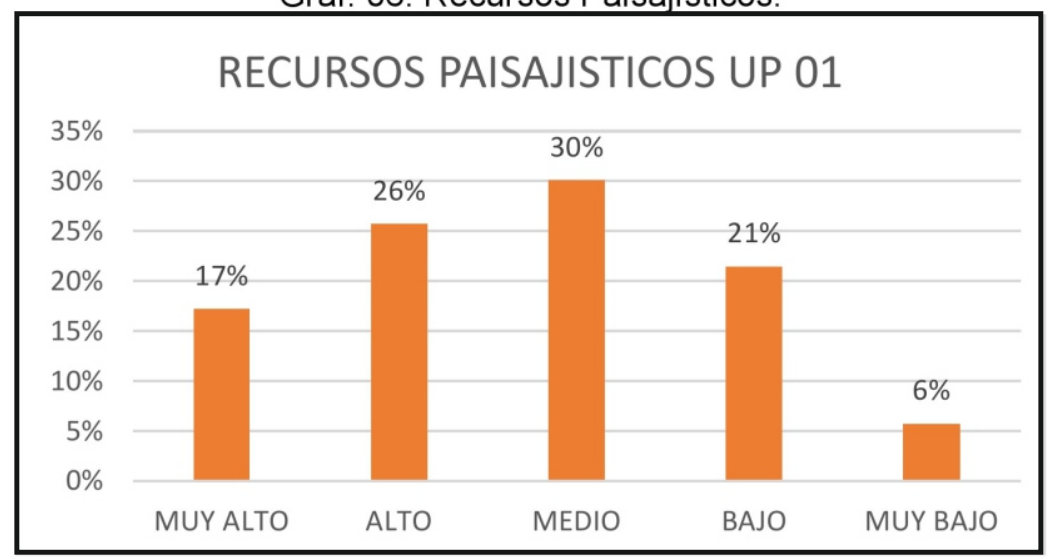

El Estado de Conservación de los componentes del paisaje de la Zona Monumental se encuentra en un $67 \%$ en bueno y el $26 \%$ la pondera en muy buen estado (Graf. 07)

Graf. 7: Análisis descriptivo de la variable Estado de Conservación

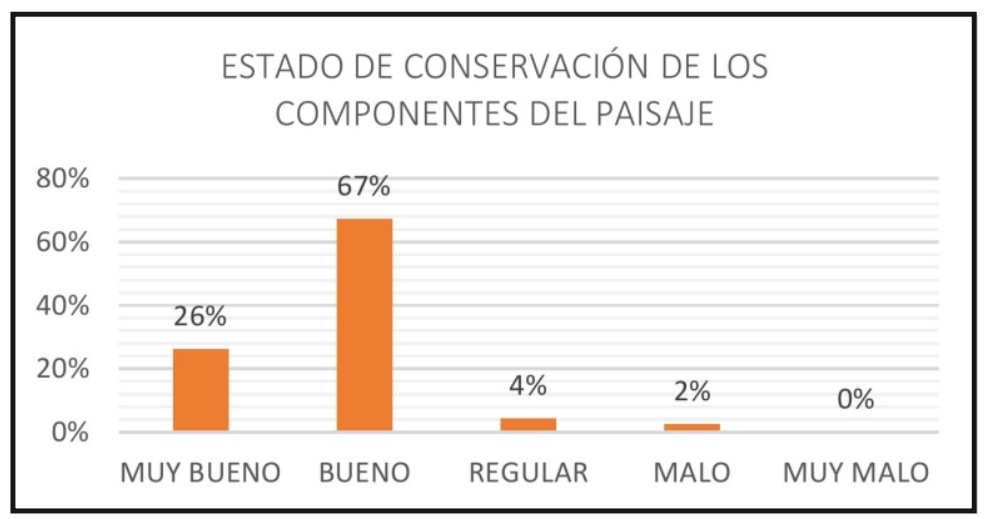

El valor de correlación de Pearson es 0,936, lo que implica que existe una relación directa significativa entre la variable valoración y estado de conservación con un nivel de significancia del 0.01 en la Zona Monumental de Tacna en el año 2016

\section{DISCUSIÓN}

Al analizar la variable dependiente "valoración del paisaje urbano", a través de tres indicadores como son: calidad visual intrínseca, puntos de observación y recursos paisajísticos, en la Zona Monumental de Tacna en el año 2016, se pudo determinar que el nivel de valoración del paisaje urbano es bueno, esta afirmación se basa en los resultados arrojados por los instrumentos aplicados durante el proceso de investigación. Lo que significa que la apreciación del usuario de la Zona Monumental de Tacna es positiva, dándole valor al conjunto urbano y paisajístico que encierra en sí misma, a la oportunidad de apreciarla desde determinados recorridos 0 sectores representativos también llamados puntos de observación y de los elementos singulares que forman parte de éste paisaje. Según la variable "estado de conservación de la Zona Monumental”, en referencia a los puntos de observación y recursos paisajísticos ha permitido observar que el nivel del estado de conservación de la Zona Monumental de Tacna en el año 2016 es bueno. Los componentes del paisaje, sumados a los puntos de observación y a los recursos paisajísticos que se ubican en el ámbito de la zona Monumental de Tacna, en su mayoría se encuentran en buen estado de conservación, encontrándose en un rango positivo. Se encontró que existe una relación directa y significativa entre la variable valoración y la variable estado de conservación en la Zona Monumental de Tacna en el año 2016 donde la variable valoración va influenciar en un $87.60 \%$ a la 
variable estado de conservación. Se recomienda realizar mayor cantidad de investigaciones en éste ámbito de estudio, ya que es poca la información documentada y existente en los repositorios que contienen información valiosa sobre la historia de la ciudad, siendo la Zona Monumental el corazón de la misma. Durante el proceso de investigación y trabajo de campo, se observó la necesidad de contar con un Manual de Diseño de Paisaje Urbano para la ciudad de Tacna, lo que permitiría ser un instrumento de difusión y de unificación de criterios para establecer pautas de diseño del paisaje urbano. La Zona Monumental de Tacna, a pesar de haber sido reducida a su mínima extensión, es un espacio urbano significativo para la ciudad de Tacna, que se fortalecería con un "Plan Concertado para la Difusión, Conservación y Mantenimiento sostenido del Paisaje Urbano de la Zona Monumental de Tacna", ya que es fundamental que el Ministerio de Cultura a través de la Dirección Desconcentrada de Cultura de Tacna y la Municipalidad Provincial de Tacna, establezcan criterios homogéneos para su conservación y mantenimiento como un paisaje urbano integral y no trabajar solamente en elementos puntuales que se consideran los más representativos, ya que el paisaje está conformado por la totalidad de sus componentes y no por elementos aislados.

\section{BIBLIOGRAFIA}

Blanco, A. A. (1979). La definición de unidades de paisaje y su clasificación en la provincia de Santander. Tesis Doctoral.

Cifuentes, P. (1979). La Calidad Visual de Unidades Territoriales. Aplicación al valle del río Tiétar. Tesis Doctoral. E.T.S. de Ing. de Montes. Universidad Politécnica, Madrid.

Comunidad Valeciana. (2016). Conselleria d'Infraestructures, Territori i Medi Ambient. Recuperado 19 de junio de 2017, a partir de http://bdb.cma.gva.es/web/acciones.aspx?url=http://b db.cma.gva.es/arbol.htm\&logo=http://bdb.cma.gva.es /bdb.jpg\&an=http://bdb.cma.gva.es/bdb2.jpg\&gana= UA-16710898-11/
Definición MX. (s. f.). Valoración. Recuperado 19 de junio de 2017, a partir de https://definicion.mx/valoracion/

Europeo, E. m. (2000). Convenio Europeo del Pisaje. Florencia.

Gómez Villarino, A. (2012). El paisaje: Diseño de una metodología para su análisis, diagnóstico, planificación e inclusión en los procesos de toma de desiciones. Tesis Doctoral. Madrid, Madrid, Espana: Universidad Pilitécnica de Madrid.

Instituto Nacional de Cultura. (2009). Glosario de téminos artísticos y museográficos (Primera ed.). Lima, Perú: Instituto Nacional de Cultura. Recuperado el 09 de enero de 2017.

Másmela Díaz, Pamela. (2010). El paisaje como elemento de la ordenación territorial. un análisis de paisaje desde su enfoque.(citado de Ocaña et al 2004).

Ministerio de Medio Ambiente y medio Rural y Marino. (2008). Plan de Participación Pública. Confederación Hidrográfica de Júcar. Provincia de Castellón.

Muñoz Criado, A. (2012). Guía Metodológica estudio de Paisaje. Valencia: Conselleria de Infraestructuras territorio y medio ambiente.

Muñoz-Pedreros, A. (2004). La evaluación del paisaje: una herramienta de gestión ambiental. Revista chilena de historia natural, 77(1), 139-156. https://doi.org/10.4067/S0716-078X2004000100011 Nogué i Font, J. (2007). La construcción social del paisaje. Cataluña: Biblioteca Nueva.

Ojeda, J., \& Cano, N. (2009). El paisaje en la memoria de los Territorios. XVII Congreso de Estudios Vascos. Ormaetxea Arenaza, O. (1997). Concepto y método en paisaje. Una propuesta docente. Lurralde -20, 333344.

Pérez, E. (2000). Paisaje Urbano en nuestras ciudades. BITACORA 4-I. Obtenido de dialnet.unirioja:

Serrano Giné, D. (2014). Unidades de paisaje naturales y unidades de paisaje artificiales. Comparación mediante SIG y métricas de paisaje. GeoFocus. Revista Internacional de Ciencia y Tecnología de la Información Geográfica, 23-54.

UNESCO. (2006). Convención del Patrimonio Mundial de UNESCO 1972. Paris: UNESCO.

Recibidos: 09 / 05 / 17

Aceptado para publicación: 09 / 06 / 17 